\title{
EL HOGAR, EL ENTORNO Y LA MORTALIDAD DE NIÑOS MENORES DE CINCO AÑOS EN BOLIVIA
}

Nicole Daniela La Ruta Rosas y Pamela Córdova Olivera

\section{RESUMEN}

A pesar de los progresos en la reducción de la mortalidad en menores de cinco años desde 1990 hasta 2015 en la región Latinoamericana, siguiendo lo planteado por los Objetivos de Desarrollo del Milenio, Bolivia es uno de los países con la mayor tasa de mortalidad solo por debajo de Haití. Identificar los determinantes de la mortalidad de la niñez, en este contexto, resulta clave para comprender el porqué del problema y su magnitud para establecer medidas de política pública correctivas que coadyuven a alcanzar los niveles de mortalidad esperados. Bajo esta premisa, la investigación emplea dos técnicas de análisis: 1) el análisis de las variables independientes sobre la condición de supervivencia del niño a través de la estadística descriptiva y 2) la aplicación de un modelo de elección binaria que permite hacer una revisión concreta acerca del efecto de cada variable independiente seleccionada sobre la probabilidad de que el niño muera antes de cumplir sesenta meses en cuanto a signo de intervención y magnitud. Los principales resultados indican que las variables que intervienen en un incremento o decremento de la probabilidad de muerte de un niño son: el tamaño al nacer, el intervalo de nacimiento precedente, el estado laboral de la madre, la presencia de lactancia materna, nivel de estudios de la madre, el número de miembros del hogar, identificación étnica de la madre y la proporción de mujeres de la comunidad que hayan tenido partos institucionales. Tales resultados muestran que la interconexión entre variables de distintos niveles: individual, hogar y contexto, es vital para explicar los niveles y tendencias de mortalidad en nuestro país.

Palabras clave: Mortalidad de la Niñez, Marco Mosley-Chen, Objetivos de Desarrollo del Milenio, Política Pública

DOI: $10.23881 /$ idupbo.017.2-4e 